\title{
NUMERICAL TREATMENT OF SINGULARLY PERTURBED FOURTH-ORDER TWO-PARAMETER PROBLEMS*
}

\author{
MIRJANA BRDAR ${ }^{\dagger}$, SEBASTIAN FRANZ ${ }^{\ddagger}$, AND HANS-GÖRG ROOS ${ }^{\S}$
}

\begin{abstract}
A singularly perturbed fourth-order problem with two small parameters is considered in one dimension and on a smooth domain in two dimensions. Using its discretisation by a mixed finite element method on a properly defined Shishkin mesh, we prove convergence in the associated energy norm.
\end{abstract} meshes

Key words. singular perturbation, fourth-order problem, two small parameters, mixed method, layer-adapted

AMS subject classifications. 65L11, 65L60, 65N30, 65N50

1. Introduction. A motivation for considering fourth-order singularly perturbed problems comes from the fact that they are currently very popular in the scientific community and have application in vibration beam problems [8] and the theory of hydrodynamic stability, like the Orr-Sommerfeld equation [6,9]. In such problems, the highest derivative in the differential equation is multiplied by a small positive parameter, which produces the appearance of layers. Standard numerical methods are inefficient for singularly perturbed problems, so the main goal in the construction of numerical methods for these problems is acquiring their uniform convergence with respect to all perturbation parameters. Recently, fourth-order singularly perturbed problems with one perturbation parameter were investigated in $[3,4,5,7,14]$.

In this paper we consider the singularly perturbed fourth-order problem with two small parameters given by

$$
\begin{aligned}
\varepsilon_{1}^{2} \Delta^{2} u-\varepsilon_{2}^{2} \Delta u+c u & =f & & \text { in } \Omega, \\
u & =0 & & \text { on } \Gamma=\partial \Omega, \\
\frac{\partial}{\partial \mathbf{n}} u & =0 & & \text { on } \Gamma=\partial \Omega,
\end{aligned}
$$

under the assumption that $c \geq c_{0}^{2}>0 \in L_{\infty}(\Omega)$ and that $0<\varepsilon_{1}<\varepsilon_{2} \ll 1$ are two small perturbation parameters. We further specify that for an arbitrary constant $C>0$

$$
\varepsilon_{1} \leq C \varepsilon_{2}^{2}
$$

as otherwise we have essentially a one-parameter singularly perturbed problem; see [6]. Note that for smooth enough functions $c$ and $f$ we have in 1D and for smooth or rectangular domains in 2D the regularity result $u \in H_{0}^{2}(\Omega) \cap H^{4}(\Omega)$; see [1].

The solution to a problem like (1.1) with perturbation parameters fulfilling (1.2) is characterised by two overlapping layers along all boundaries. In Section 2 we analyse the structure in $1 \mathrm{D}$ in more detail and present a numerical method for simulating the problem. In Section 3 we consider smooth domains $\Omega \subset \mathbb{R}^{2}$ and present a numerical analysis for our numerical method. Section 4 then gives some examples and results supporting our theoretical findings.

* Received March 19, 2018. Accepted December 18, 2018. Published online on March 6, 2019. Recommended by Bill Layton.

${ }^{\dagger}$ Faculty of Technology, University of Novi Sad, Serbia (mirjana.brdar@uns.ac.rs).

$\ddagger$ Institute of Scientific Computing, Technische Universität Dresden, Germany (sebastian.franzetu-dresden.de).

${ }^{\S}$ Institute of Numerical Mathematics, Technische Universität Dresden, Germany (hans-goerg.roosetu-dresden.de). 
1.1. Notation. We will use $\|\cdot\|_{k, D}$ to denote the $H^{k}$-norm and $\|\cdot\|_{L^{p}(D)}$ to denote the $L^{p}$-norm on $D \subset \Omega$. If $D=\Omega$, then the reference to the domain may be suppressed. For the $L^{2}$-scalar product in $\Omega$ we write $\langle\cdot, \cdot\rangle$. Furthermore, we present estimates using $A \lesssim B$ if a generic constant $C$ independent of $\varepsilon_{1}, \varepsilon_{2}$ and the numerical method parameter $N$ exists such that $A \leq C B$. For $A \lesssim B$ and $B \lesssim A$, we write shortly $A \sim B$.

2. Analysis in 1D. For this section let $\Omega=(0,1)$ be the unit interval in 1D. If we want to construct any numerical method for solving singularly perturbed problem in a uniform way, then it is crucial to have information about the behaviour of the derivatives of the exact solution. The bounds for the derivatives are required in the mesh definition as well as in the error analysis. The behaviour of the solution for the problem (1.1) in 1D can be deduced from the asymptotic expansion of [6]. Here, we are only interested in the case when $\varepsilon_{1} \ll \varepsilon_{2}^{2}$, as in the cases of $\varepsilon_{1}=\alpha \varepsilon_{2}^{2}$ and $\varepsilon_{1} \gg \varepsilon_{2}^{2}$, we have essentially singularly perturbed problems with one perturbation parameter.

ASSUMPTION 2.1. The solution u of the boundary value problem (1.1) in $1 D$ can be decomposed as

$$
u=S+E_{1}+E_{2}+E_{3}+E_{4},
$$

where $S$ is the smooth part of the solution, $E_{1}$ and $E_{2}$ are layer parts near $x=0$, and $E_{3}$ and $E_{4}$ are layer parts near $x=1$. We assume that for $0 \leq k \leq 4$,

$$
\left|S^{(k)}(x)\right| \lesssim 1, \quad\left|E_{1}^{(k)}(x)\right| \lesssim \frac{1}{\varepsilon_{2}}\left(\frac{\varepsilon_{1}}{\varepsilon_{2}}\right)^{1-k} \mathrm{e}^{-\frac{x \varepsilon_{2}}{\varepsilon_{1}}}, \quad\left|E_{2}^{(k)}(x)\right| \lesssim \varepsilon_{2}^{-k} \mathrm{e}^{-\frac{x}{\varepsilon_{2}}},
$$

for $x \in \Omega$ and similarly for $E_{3}$ and $E_{4}$ with $x$ being replaced by $1-x$ in the bounds.

With this assumption on the solution decomposition, we introduce a layer-adapted mesh based on the standard Shishkin mesh $[11,12]$. Let the number $N$ be a positive integer divisible by 8 , and let

$$
\lambda_{1}=\min \left\{\sigma_{1} \frac{\varepsilon_{1}}{\varepsilon_{2}} \ln N, \frac{1}{8}\right\}, \quad \lambda_{2}=\min \left\{\sigma_{2} \varepsilon_{2} \ln N, \frac{1}{4}\right\}
$$

where $\sigma_{i}, i=1,2$, are user-chosen parameters. Then we define a mesh on $\bar{\Omega}=[0,1]$ constructed with five distinct uniform meshes separated by transition points located at $\lambda_{1}, \lambda_{2}$, $1-\lambda_{2}$, and $1-\lambda_{1}$. Each of the subintervals

$$
\Omega_{1}=\left[0, \lambda_{1}\right], \quad \Omega_{2}=\left[\lambda_{1}, \lambda_{2}\right], \quad \Omega_{4}=\left[1-\lambda_{2}, 1-\lambda_{1}\right], \quad \Omega_{5}=\left[1-\lambda_{1}, 1\right]
$$

is divided into $N / 8$ equal mesh elements, and the subinterval $\Omega_{3}=\left[\lambda_{2}, 1-\lambda_{2}\right]$ is divided into $N / 2$ equal mesh elements.

Let us denote the length of a cell in $\Omega_{1} \cup \Omega_{5}$ by $h_{1}$, of a cell in $\Omega_{2} \cup \Omega_{4}$ by $h_{2}$, and in $\Omega_{3}$ by $h_{3}$. For them we have

$$
\begin{aligned}
& h_{1}=\frac{8 \lambda_{1}}{N} \sim \frac{\varepsilon_{1}}{\varepsilon_{2}} N^{-1} \ln N, \\
& h_{2}=\frac{8\left(\lambda_{2}-\lambda_{1}\right)}{N} \sim \varepsilon_{2} N^{-1} \ln N, \\
& h_{3}=\frac{2\left(1-2 \lambda_{2}\right)}{N} \sim N^{-1} .
\end{aligned}
$$


Following the idea from [4], we rewrite the problem (1.1) as a system using the auxiliary unknown $w=\varepsilon_{1} u^{\prime \prime} \in H^{2}(\Omega)$. Then a standard weak formulation for (1.1) is as follows: find $(u, w) \in H_{0}^{1}(\Omega) \times H^{1}(\Omega)$ such that

$$
\begin{aligned}
\varepsilon_{1}\left\langle u^{\prime}, \varphi^{\prime}\right\rangle+\langle w, \varphi\rangle & =0, & & \forall \varphi \in H^{1}(\Omega), \\
\varepsilon_{2}^{2}\left\langle u^{\prime}, \psi^{\prime}\right\rangle+\langle c u, \psi\rangle-\varepsilon_{1}\left\langle w^{\prime}, \psi^{\prime}\right\rangle & =\langle f, \psi\rangle, & & \forall \psi \in H_{0}^{1}(\Omega) .
\end{aligned}
$$

By using the bilinear form

$$
a((u, w),(\psi, \varphi)):=\langle w, \varphi\rangle+\varepsilon_{2}^{2}\left\langle u^{\prime}, \psi^{\prime}\right\rangle+\langle c u, \psi\rangle+\varepsilon_{1}\left(\left\langle u^{\prime}, \varphi^{\prime}\right\rangle-\left\langle w^{\prime}, \psi^{\prime}\right\rangle\right),
$$

we can rewrite the task as: find $(u, w) \in H_{0}^{1}(\Omega) \times H^{1}(\Omega)$ such that

$$
a((u, w),(\psi, \varphi))=\langle f, \psi\rangle, \quad \forall(\psi, \varphi) \in H_{0}^{1}(\Omega) \times H^{1}(\Omega) .
$$

Let $S_{h}^{0}=\left\{v \in H_{0}^{1}(\Omega):\left.v\right|_{\tau} \in \mathcal{P}_{1}(\tau)\right\}$ and $S_{h}=\left\{v \in H^{1}(\Omega):\left.v\right|_{\tau} \in \mathcal{P}_{1}(\tau)\right\}$ be the finite element spaces of piecewise linear functions defined on the Shishkin mesh. The discrete problem is then characterised by: find $\left(u_{N}, w_{N}\right) \in S_{h}^{0} \times S_{h}$ such that

$$
a\left(\left(u_{N}, w_{N}\right),(\psi, \varphi)\right)=\langle f, \psi\rangle, \quad \forall \varphi \in S_{h}, \psi \in S_{h}^{0} .
$$

The bilinear form is coercive with respect to the energy norm given by

$$
\|(u, w)\|^{2}:=\|w\|_{0}^{2}+\varepsilon_{2}^{2}\left\|u^{\prime}\right\|_{0}^{2}+\left\|c^{1 / 2} u\right\|_{0}^{2} .
$$

Hence, the standard weak formulation and the discretisation method have unique solutions.

In order to estimate interpolation errors, we use standard piecewise linear interpolants $I: C(\Omega) \rightarrow V_{0}(\Omega)$ and $J: C(\Omega) \rightarrow V(\Omega)$ defined by interpolating the values at the mesh nodes.

LEMMA 2.2. For $\sigma_{1} \geq 2$ and $\sigma_{2} \geq 2$, we have

$$
\|u-I u\|_{0}+\|w-J w\|_{0} \lesssim N^{-2} \ln ^{3 / 2} N, \quad\left\|(u-I u)^{\prime}\right\|_{0} \lesssim \varepsilon_{2}^{-1 / 2} N^{-1} \ln N .
$$

Proof. For the smooth part $S$ of the solution $u$ we have by standard interpolation error estimates on each subinterval

$$
\|S-I S\|_{0} \lesssim\left(h_{1}^{2}+h_{2}^{2}+h_{3}^{2}\right)\left\|S^{\prime \prime}\right\|_{0} \lesssim N^{-2} .
$$

Similarly, we have for the first layer part $E^{1}=E_{1}+E_{3}$,

$$
\begin{aligned}
&\left\|E^{1}-I E^{1}\right\|_{0, \Omega_{1} \cup \Omega_{5}} \lesssim h_{1}^{2}\left\|E^{1^{\prime \prime}}\right\|_{0, \Omega_{1} \cup \Omega_{5}} \lesssim\left(\frac{\varepsilon_{1}}{\varepsilon_{2}}\right)^{1 / 2}\left(N^{-1} \ln N\right)^{2}, \\
&\left\|E^{1}-I E^{1}\right\|_{0, \Omega_{2} \cup \Omega_{3} \cup \Omega_{4}}^{2} \lesssim\left\|E^{1}\right\|_{L^{\infty}\left(\Omega_{2} \cup \Omega_{3} \cup \Omega_{4}\right)} \lesssim N^{-\sigma_{1}} \lesssim N^{-2},
\end{aligned}
$$

where the $L^{\infty}$-stability of the interpolation and $\sigma_{1} \geq 2$ was used. For the second layer part $E^{2}=E_{2}+E_{4}$, we have similarly

$$
\begin{aligned}
\left\|E^{2}-I E^{2}\right\|_{0, \Omega_{1} \cup \Omega_{2} \cup \Omega_{4} \cup \Omega_{5}} & \lesssim\left(h_{1}^{2}+h_{2}^{2}\right)\left\|E^{2^{\prime \prime}}\right\|_{0, \Omega_{1} \cup \Omega_{2} \cup \Omega_{4} \cup \Omega_{5}} \\
& \lesssim\left(\frac{\varepsilon_{1}}{\varepsilon_{2}}+\varepsilon_{2}\right)^{1 / 2}\left(N^{-1} \ln N\right)^{2}, \\
\left\|E^{2}-I E^{2}\right\|_{0, \Omega_{3}}^{2} & \lesssim\left\|E^{2}\right\|_{L^{\infty}\left(\Omega_{3}\right)} \lesssim N^{-\sigma_{2}} \lesssim N^{-2},
\end{aligned}
$$


now using $\sigma_{2} \geq 2$. Combining these estimates with $\varepsilon_{2} \ln N \lesssim 1$ and $\frac{\varepsilon_{1}}{\varepsilon_{2}} \ln N \lesssim 1$, we obtain the first result. The results for $w=\varepsilon_{1} u^{\prime \prime}$ follow in the same way.

For the $H^{1}$-error we use again the above techniques, now for the derivative, and a trick from [13, proof of Lemma 3.2] to estimate $\|I E\|_{0, \Omega_{2} \cup \Omega_{4}}$ in an optimal way.

Having the interpolation error bound, we only need estimates for the discrete error $I u-u_{N}$.

THEOREM 2.3. For the discrete error on a Shishkin mesh with $\sigma_{1}, \sigma_{2} \geq 2$, where $\left(u_{N}, w_{N}\right)$ is the solution of (2.2), we have the supercloseness estimate

$$
\left\|\left(I u-u_{N}, J w-w_{N}\right)\right\| \lesssim N^{-2}(\ln N)^{3 / 2} .
$$

Proof. Let $\psi=I u-u_{N} \in V_{N}^{0}$ and $\varphi=J w-w_{N} \in V_{N}$. Starting with the coercivity and Galerkin orthogonality of the bilinear form $a((\cdot, \cdot),(\cdot, \cdot))$, we have

$$
\begin{aligned}
\|(\psi, \varphi)\|^{2} \leq & a((I u-u, J w-w),(\psi, \varphi)) \\
= & \langle J w-w, \varphi\rangle+\varepsilon_{2}^{2}\left\langle(I u-u)^{\prime}, \psi^{\prime}\right\rangle+\langle c(I u-u), \psi\rangle \\
& \quad+\varepsilon_{1}\left(\left\langle(I u-u)^{\prime}, \varphi^{\prime}\right\rangle-\left\langle(J w-w)^{\prime}, \psi^{\prime}\right\rangle\right) \\
= & I_{1}+I_{2}+I_{3}+I_{4}+I_{5} .
\end{aligned}
$$

By definition of the interpolation operators the quantities $I u-u$ and $J w-w$ are zero at the mesh points. Therefore, $I_{2}, I_{4}, I_{5}$ are also zero. With the Cauchy-Schwarz inequality and Lemma 2.2, we obtain for the remaining two terms

$$
\begin{aligned}
& \left|I_{1}\right| \leq\|J w-w\|_{0}\|\varphi\|_{0} \lesssim N^{-2} \ln ^{3 / 2} N\|\varphi\|_{0} \\
& \left|I_{3}\right| \leq\|I u-u\|_{0}\|\psi\|_{0} \lesssim N^{-2} \ln ^{3 / 2} N\|\psi\|_{0}
\end{aligned}
$$

Combining the estimates for the interpolation error with those for the discrete error we obtain the main result of this section.

THEOREM 2.4. On a Shishkin mesh with $\sigma_{1}, \sigma_{2} \geq 2$, where $(u, w)$ is the solution of (2.1) and $\left(u_{N}, w_{N}\right)$ is the solution of (2.2), the following estimate holds:

$$
\left\|\left(u-u_{N}, w-w_{N}\right)\right\| \| N^{-2}(\ln N)^{3 / 2}+\varepsilon_{2}^{1 / 2} N^{-1} \ln N .
$$

3. Smooth domains in 2D. Let us now assume that $\Omega$ is the interior of a smooth, nonoverlapping curve $\Gamma=\partial \Omega$.

3.1. Decomposition of solution and domain. We will dissect $\Omega$ into three disjoint subdomains that are then discretised. We follow the idea by Shishkin and define for given mesh-parameters $N, \sigma_{1}, \sigma_{2}$ the transition points

$$
\lambda_{1}=\sigma_{1} \frac{\varepsilon_{1}}{\varepsilon_{2}} \ln N, \quad \lambda_{2}=\sigma_{2} \varepsilon_{2} \ln N .
$$

We will give precise bounds for the user-chosen parameters $\sigma_{1}, \sigma_{2}$ later. The parameter $N$ is used to parameterise the number of cells in the final mesh (and thus the number of unknowns).

Like in the 1D-case we assume $\varepsilon_{1} \ll \varepsilon_{2}^{2}$ and $\varepsilon_{2}$ to be small enough such that $\lambda_{1} \leq \frac{1}{2} \lambda_{2} \lesssim 1$ holds as otherwise no special treatment of the boundary layers is needed. Furthermore, the above assumption guarantees that the numerical layer further away from the boundary is not smaller than the numerical layer near the boundary. 
With the distance $\rho=d(\mathbf{x}, \Gamma)$ of a point $\mathbf{x}$ to the boundary $\Gamma$ and $\theta$ as the local coordinates near the boundary, we define

$$
\begin{aligned}
& \Omega_{1}:=\left\{\mathbf{x} \in \Omega: d(\mathbf{x}, \Gamma)<\lambda_{1}\right\}, \\
& \Omega_{2}:=\left\{\mathbf{x} \in \Omega: \lambda_{1}<d(\mathbf{x}, \Gamma)<\lambda_{2}\right\}, \quad \text { and } \\
& \Omega_{3}:=\left\{\mathbf{x} \in \Omega: \lambda_{2}<d(\mathbf{x}, \Gamma)\right\} .
\end{aligned}
$$

The reason for choosing the transition parameters as above is inspired by the 1D-analysis. We assume a similar decomposition for the smooth-domain case. Then the reasoning of Shishkin's approach is to define the transition points such that the sizes of the exponentially decaying boundary-layer terms are as small as $N^{-\sigma}$ at $\lambda$. The precise assumptions needed for our analysis are given in the following.

ASSUMPTION 3.1. Let us assume that $u$ and $w=\varepsilon_{1} \Delta u$ can be decomposed as

$$
u=S+E_{1}+E_{2} \quad \text { and } \quad w=\tilde{S}+\tilde{E}_{1}+\tilde{E}_{2},
$$

where we have the estimates

$$
\begin{gathered}
\|S\|_{3} \lesssim 1, \quad\|\tilde{S}\|_{3} \lesssim \varepsilon_{1}, \\
\left\|E_{1}\right\|_{L^{\infty}\left(\Omega_{3}\right)}+\varepsilon_{2}^{1 / 2}\left\|E_{1}\right\|_{1, \Omega_{3}}+\left\|\tilde{E}_{1}\right\|_{L^{\infty}\left(\Omega_{3}\right)}+\varepsilon_{2}^{1 / 2}\left\|\tilde{E}_{1}\right\|_{1, \Omega_{3}} \lesssim \mathrm{e}^{-\frac{\lambda_{1}}{\varepsilon_{1} / \varepsilon_{2}}} \lesssim N^{-\sigma_{1}}, \\
\left\|E_{2}\right\|_{L^{\infty}\left(\Omega_{3}\right)}+\varepsilon_{2}^{1 / 2}\left\|E_{2}\right\|_{1, \Omega_{3}}+\left\|\tilde{E}_{2}\right\|_{L^{\infty}\left(\Omega_{3}\right)}+\varepsilon_{2}^{1 / 2}\left\|\tilde{E}_{2}\right\|_{1, \Omega_{3}} \lesssim \mathrm{e}^{-\frac{\lambda_{2}}{\varepsilon_{2}}} \lesssim N^{-\sigma_{2}},
\end{gathered}
$$

and the pointwise bounds, for $0 \leq \rho \leq \lambda_{2}$ and $0 \leq \ell+k \leq 3$,

$$
\left|\partial_{\theta}^{\ell} \partial_{\rho}^{k} E_{1}(\rho, \theta)\right| \lesssim \frac{1}{\varepsilon_{2}}\left(\frac{\varepsilon_{1}}{\varepsilon_{2}}\right)^{1-k} \mathrm{e}^{-\frac{\rho}{\varepsilon_{1} / \varepsilon_{2}}}, \quad\left|\partial_{\theta}^{\ell} \partial_{\rho}^{k} E_{2}(\rho, \theta)\right| \lesssim \varepsilon_{2}^{-k} \mathrm{e}^{-\frac{\rho}{\varepsilon_{2}}},
$$

respectively, for $0 \leq \ell+k \leq 2$,

$$
\left|\partial_{\theta}^{\ell} \partial_{\rho}^{k} \tilde{E}_{1}(\rho, \theta)\right| \lesssim\left(\frac{\varepsilon_{1}}{\varepsilon_{2}}\right)^{-k} \mathrm{e}^{-\frac{\rho}{\varepsilon_{1} / \varepsilon_{2}}}, \quad\left|\partial_{\theta}^{\ell} \partial_{\rho}^{k} \tilde{E}_{2}(\rho, \theta)\right| \lesssim \varepsilon_{2}^{-k} \mathrm{e}^{-\frac{\rho}{\varepsilon_{2}}}
$$

REMARK 3.2. The above assumptions are reasonable. If we consider the decomposition of the 1D-example and use it as an approximation near the (stretched) boundary, then the above stated assumptions follow. Furthermore, for the case $\varepsilon_{2}=1$, they correspond well with those in [2] on smooth domains and those of [4, 5] on a square-domain.

REMARK 3.3. By assuming $\partial \Omega$ to be smooth, the first derivatives with respect to the (physical) $x, y$ variables are bounded by the first derivatives with respect to the $\rho, \theta$ variables.

3.2. Definition of the mesh. Figure 3.1 shows exemplarily a mesh for a smooth domain that is also used in the numerical examples. We use mapped quadrilaterals to discretise the layer regions $\Omega_{1}$ (between the two outer thick lines in Figure 3.1) and $\Omega_{2}$ (between the two inner thick lines). The mapping is defined such that the boundary is exactly matched using the ideas from [10] — there is no approximation of the curved boundary. In $\Omega_{1}$ and $\Omega_{2}$ we place $\frac{N}{8}$ plies of mapped quadrilaterals in such a way that they are of the same size in $\rho$-direction and thereby giving the analogon of the 1D-Shishkin mesh. For the domain $\Omega_{3}$ we can use either mapped triangles or quadrilaterals to discretise it. Here we use triangles. Again there is no approximation of the curved transition line between $\Omega_{2}$ and $\Omega_{3}$. Depending on the given setting, all cells in $\Omega_{3}$ are of similar size, and the mesh in $\Omega_{3}$ is quasi-uniform. The number of cells in $\Omega_{3}$ is proportional to $N^{2}$, and therefore the size of a cell $\tau \subset \Omega_{3}$ can be estimated by

$$
\operatorname{diam}(\tau) \sim H=\frac{1}{N}
$$




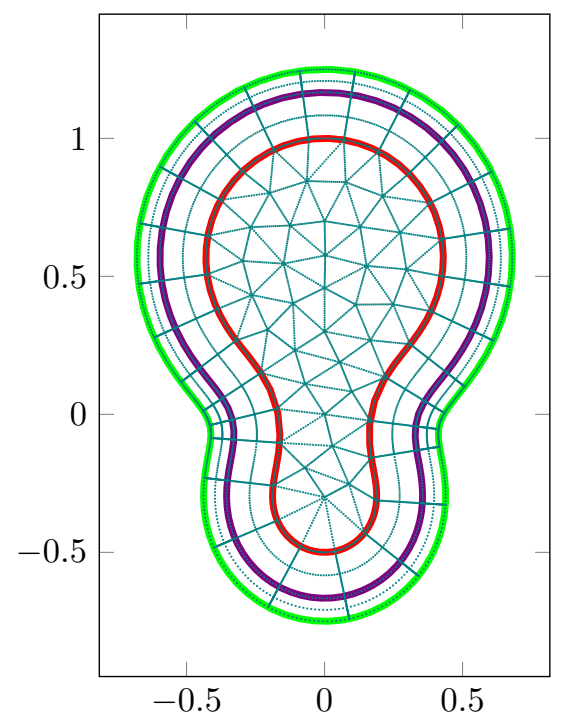

FIG. 3.1. Mesh of a smooth domain with $N=16$.

For the cells $\tau$ in the layer region with the corresponding cells $\hat{\tau}$ in the reference domain, the dimensions $h_{1}$ by $k_{1}$ in $\Omega_{1}$ and $h_{2}$ by $k_{2}$ in $\Omega_{2}$ follow, where

$$
h_{1}, h_{2} \sim H, \quad k_{1} \sim \frac{\lambda_{1}}{N} \sim \frac{\varepsilon_{1}}{\varepsilon_{2}} N^{-1} \ln N, \quad \text { and } \quad k_{2} \sim \frac{\lambda_{2}}{N} \sim \varepsilon_{2} N^{-1} \ln N .
$$

3.3. Numerical method. Similarly to the $1 \mathrm{D}$ case we rewrite the fourth-order equation as a system of two second-order equations for $u \in H_{0}^{1}(\Omega)$ and $w=\varepsilon_{1} \Delta u \in H^{1}(\Omega)$ :

$$
\begin{aligned}
-\varepsilon_{1} \Delta u+w & =0, \\
\varepsilon_{1} \Delta w-\varepsilon_{2}^{2} \Delta u+c u & =f .
\end{aligned}
$$

Its weak formulation now reads: find $(u, w) \in H_{0}^{1}(\Omega) \times H^{1}(\Omega)$ such that

$$
\begin{aligned}
a((u, w),(\psi, \varphi)):=\langle w, \varphi\rangle & +\varepsilon_{2}^{2}\langle\nabla u, \nabla \psi\rangle+\langle c u, \psi\rangle \\
& +\varepsilon_{1}\langle\nabla u, \nabla \varphi\rangle-\varepsilon_{1}\langle\nabla w, \nabla \psi\rangle=\langle f, \psi\rangle
\end{aligned}
$$

for all $(\psi, \varphi) \in H_{0}^{1}(\Omega) \times H^{1}(\Omega)$.

Our discrete space is piecewise polynomial in the reference domain. For any cell $\tau \subset \Omega$, let $m_{\tau}$ be the mapping from one of the three reference domains into the mesh on $\Omega$. Then our first discrete space is given by

$$
\begin{aligned}
V_{N}:=\left\{v \in H^{1}(\Omega):\left.v\right|_{\tau}=\hat{v}_{\hat{\tau}} \circ m_{\tau}^{-1}, \hat{v}_{\hat{\tau}}\right. & \in \mathcal{Q}_{1}(\hat{\tau}) \text { for } \tau \subset \Omega_{1} \cup \Omega_{2}, \\
\hat{v}_{\hat{\tau}} & \left.\in \mathcal{P}_{1}(\hat{\tau}) \text { for } \tau \subset \Omega_{3}\right\} .
\end{aligned}
$$

The second discrete space is simply

$$
V_{N}^{0}:=V_{N} \cap H_{0}^{1}(\Omega) .
$$


Our discrete problem now reads: find $\left(u_{N}, w_{N}\right) \in V_{N}^{0} \times V_{N}$ such that

$$
a\left(\left(u_{N}, w_{N}\right),(\psi, \varphi)\right)=\langle f, \psi\rangle
$$

for all $(\psi, \varphi) \in V_{N}^{0} \times V_{N}$.

REMARK 3.4. By definition we immediately have Galerkin orthogonality

$$
a\left(\left(u-u_{N}, w-w_{N}\right),(\psi, \varphi)\right)=0
$$

and coercivity

$$
a((u, w),(u, w)) \geq\|w\|_{0}^{2}+\varepsilon_{2}^{2}\|\nabla u\|_{0}^{2}+\left\|c_{0} u\right\|_{0}^{2}=:|\|(u, w)\||^{2}
$$

in the energy norm. Note that we have with $w=\varepsilon_{1} \Delta u$

$$
\|(u, w) \mid\|^{2}=\varepsilon_{1}^{2}\|\Delta u\|_{0}^{2}+\varepsilon_{2}^{2}\|\nabla u\|_{0}^{2}+\left\|c_{0} u\right\|_{0}^{2} .
$$

Thus the energy norm is equivalent to the classical energy norm for the problem (1.1).

3.4. Analysis of the method. The convergence analysis follows the standard procedure of estimating the interpolation error in various norms and then estimating the discrete error by the interpolation error in the bilinear form. Let us start by defining suitable interpolation operators. Here we use the canonical vertex-interpolation operators

$$
I: C(\Omega) \rightarrow V_{N}^{0} \quad \text { and } \quad J: C(\Omega) \rightarrow V_{N}
$$

defined by point-evaluation in the parameter space. Note that we have for a quadrilateral cell $\tau$ the estimates

$$
\begin{aligned}
\|u-I u\|_{0, \tau} & \lesssim h_{\hat{\tau}}^{2}\left\|\partial_{\theta}^{2} \hat{u}\right\|_{0, \hat{\tau}}+k_{\hat{\tau}}^{2}\left\|\partial_{\rho}^{2} \hat{u}\right\|_{0, \hat{\tau}} \\
\|u-I u\|_{1, \tau} & \lesssim h_{\hat{\tau}}\left\|\partial_{\theta}^{2} \hat{u}\right\|_{0, \hat{\tau}}+\left(k_{\hat{\tau}}+h_{\hat{\tau}}\right)\left\|\partial_{\theta} \partial_{\rho} \hat{u}\right\|_{0, \hat{\tau}}+k_{\hat{\tau}}\left\|\partial_{\rho}^{2} \hat{u}\right\|_{0, \hat{\tau}}
\end{aligned}
$$

and similarly for $w-J w$ because of the transformation between real-world and parameter space.

Lemma 3.5. Let $(u, w)$ be the solution of (3.1) fulfilling Assumption 3.1. Then under the conditions $\sigma_{1} \geq 2$ and $\sigma_{2} \geq 2$, we have the estimates for the interpolation error

$$
\begin{aligned}
\|u-I u\|_{0}+\|w-J w\|_{0} & \lesssim N^{-2}(\ln N)^{3 / 2}, \\
\|u-I u\|_{1} & \lesssim \varepsilon_{2}^{-1 / 2} N^{-1} \ln N, \\
\|w-J w\|_{1} & \lesssim\left(\frac{\varepsilon_{1}}{\varepsilon_{2}}\right)^{-1 / 2} N^{-1} \ln N, \\
\|E-I E\|_{1, \Omega_{3}} & \lesssim \varepsilon_{2}^{-1 / 2} N^{-\left(\sigma_{2}-1\right)} \ln N,
\end{aligned}
$$

where $E$ is any of the boundary layer terms.

Proof. The estimation follows the standard procedure of evaluating each component of the solution decomposition on each part of the mesh separately by either using the anisotropic interpolation error formulas (3.3) if the resulting derivatives can be bounded by the mesh-sizes or by the stability of the interpolation with respect to the $L^{\infty}$ - or $W^{1, \infty}$-norms and inverse inequalities if the solution component is already small enough. Therefore, we skip the details here. 
Let us now consider estimating the discrete error

$$
(\psi, \varphi):=\left(I u-u_{N}, J w-w_{N}\right) \in V_{N}^{0} \times V_{N} .
$$

Using coercivity and Galerkin-orthogonality we have

$$
\begin{aligned}
&\|(\psi, \varphi)\|^{2} \leq a((I u-u, J w-w),(\psi, \varphi)) \\
&=\langle J w-w, \varphi\rangle+\varepsilon_{2}^{2}\langle\nabla(I u-u), \nabla \psi\rangle+\langle c(I u-u), \psi\rangle \\
& \quad+\varepsilon_{1}\langle\nabla(I u-u), \nabla \varphi\rangle-\varepsilon_{1}\langle\nabla(J w-w), \nabla \psi\rangle \\
&=: I+I I+I I I+I V+V .
\end{aligned}
$$

In contrast to the $1 \mathrm{D}$ case, all terms have to be estimated as none is vanishing.

LEMMA 3.6. Let $(u, w)$ be the solution of (3.1) fulfilling Assumption 3.1 and $\left(u_{N}, w_{N}\right)$ be the solution of (3.2). Under the conditions $\sigma_{1} \geq 2$ and $\sigma_{2} \geq 2$, we have

$$
\begin{aligned}
|I|+|I I I| & \lesssim N^{-2}(\ln N)^{3 / 2}|\|(\psi, \varphi)\|| \\
|I I| & \lesssim \varepsilon_{2}^{1 / 2} N^{-1} \ln N|\|(\psi, \varphi)\|| \\
|V| & \lesssim\left(\frac{\varepsilon_{1}}{\varepsilon_{2}}\right)^{1 / 2} N^{-1} \ln N\||(\psi, \varphi)|\| .
\end{aligned}
$$

Proof. The first bound follows directly from the Cauchy-Schwarz inequality and (3.4). Similarly the other two estimates follow using (3.5) and (3.6)

$$
\begin{aligned}
& |I I| \lesssim \varepsilon_{2}^{2}\|I u-u\|_{1}\|\psi\|_{1} \lesssim \varepsilon_{2}^{1 / 2} N^{-1} \ln N\left(\varepsilon_{2}\|\psi\|_{1}\right) \lesssim \varepsilon_{2}^{1 / 2} N^{-1} \ln N\|(\psi, \varphi)\| \| \\
& |V| \lesssim \varepsilon_{1}\|J w-w\|_{1}\|\psi\|_{1} \lesssim\left(\frac{\varepsilon_{1}}{\varepsilon_{2}}\right)^{1 / 2} N^{-1} \ln N\|(\psi, \varphi)\|
\end{aligned}
$$

The only term left is $I V$. A direct estimation as above is not applicable as $\|\nabla \varphi\|_{0}$ is not part of the energy norm. Thus we have to bound this term more elaborately and under stronger conditions.

LEMma 3.7. Let $(u, w)$ be the solution of (3.1) fulfilling Assumption 3.1 and $\left(u_{N}, w_{N}\right)$ be the solution of (3.2). Under the conditions $\sigma_{1} \geq 2, \sigma_{2} \geq 2+\frac{1}{4}$, and $\varepsilon_{1} \lesssim N^{-1}$, we have

$$
|I V| \lesssim N^{-1} \ln N\|\varphi\|_{0}
$$

Proof. The proof consists of two parts. We use sharper estimates for the interpolation error in the coarse (triangulated) domain $\Omega_{3}$ and supercloseness formulas in the remaining domain.

1) By (3.7) for the boundary layer components and standard interpolation error estimates for the smooth component $S$ of the solution decomposition, we obtain

$$
\begin{aligned}
\varepsilon_{1}\left|\langle\nabla(I u-u), \nabla \varphi\rangle_{\Omega_{3}}\right| & \lesssim \varepsilon_{1}\left(N^{-1}\|S\|_{2, \Omega_{3}}+\varepsilon_{2}^{-1 / 2} N^{-\left(\sigma_{2}-1\right)} \ln N\right) N\|\varphi\|_{0, \Omega_{3}} \\
& \lesssim\left(\varepsilon_{1}+\varepsilon_{1}^{3 / 4} N^{-\left(\sigma_{2}-2\right)} \ln N\right)\|\varphi\|_{0} \\
& \lesssim N^{-1} \ln N\|\varphi\|_{0},
\end{aligned}
$$

where the stronger conditions on $\varepsilon_{1}$ and $\sigma_{2}$ were used. 
2) For the other term we use supercloseness formulas; see, e.g., [15]. For them let $v$ be defined on the rectangular cell $\tau$ with sides of length $h_{\tau}$ and $k_{\tau}$ in an arbitrary $s$ - and $t$-direction and on a rectangular domain $T$ consisting of cells $\tau$ with sides $\ell_{1}$ and $\ell_{2}$ for minimal and maximal $t$. Then we have

$$
\begin{aligned}
\left|\left\langle\partial_{s}(I v-v), \partial_{s} \varphi\right\rangle_{\tau}\right| \lesssim & k_{\tau}^{2}\left\|\partial_{s} \partial_{t}^{2} v\right\|_{L^{\infty}(\tau)}\left\|\partial_{s} \varphi\right\|_{L^{1}(\tau)}, \\
\left|\left\langle\partial_{s}(I v-v), \partial_{t} \varphi\right\rangle_{T}\right| \lesssim & \sum_{\tau \subset T}\left(k_{\tau}\left\|\partial_{s} \partial_{t}^{2} v\right\|_{L^{\infty}(\tau)}+h_{\tau}\left\|\partial_{s}^{2} \partial_{t} v\right\|_{L^{\infty}(\tau)}\right)\|\varphi\|_{L^{1}(\tau)} \\
& \quad+\sum_{i=1}^{2} \sum_{\substack{\tau \subset T \\
\tau \cap \ell_{i} \neq \emptyset}} h_{\tau}\left\|\partial_{s}^{2} v\right\|_{L^{\infty}\left(\ell_{i} \cap \partial \tau\right)}\|\varphi\|_{L^{1}\left(\ell_{i} \cap \partial \tau\right)} .
\end{aligned}
$$

The second sum in (3.8b) can be set to zero if $\left.v\right|_{\ell_{1}}=\left.v\right|_{\ell_{2}}$.

Now let us look at the remaining term

$$
\begin{aligned}
\varepsilon_{1}\left|\langle\nabla(I u-u), \nabla \varphi\rangle_{\Omega_{1} \cup \Omega_{2}}\right| \lesssim \varepsilon_{1} & \left|\left\langle\partial_{\theta}(\hat{I} \hat{u}-\hat{u}), \partial_{\theta} \hat{\varphi}\right\rangle_{\hat{\Omega}_{1} \cup \hat{\Omega}_{2}}\right| \\
& +\varepsilon_{1}\left|\left\langle\partial_{\rho}(\hat{I} \hat{u}-\hat{u}), \partial_{\rho} \hat{\varphi}\right\rangle_{\hat{\Omega}_{1} \cup \hat{\Omega}_{2}}\right| \\
& +\varepsilon_{1}\left|\left\langle\partial_{\theta}(\hat{I} \hat{u}-\hat{u}), \partial_{\rho} \hat{\varphi}\right\rangle_{\hat{\Omega}_{1} \cup \hat{\Omega}_{2}}\right| \\
& +\varepsilon_{1}\left|\left\langle\partial_{\rho}(\hat{I} \hat{u}-\hat{u}), \partial_{\theta} \hat{\varphi}\right\rangle_{\hat{\Omega}_{1} \cup \hat{\Omega}_{2}}\right| \\
=: & a+b+c+d .
\end{aligned}
$$

We use (3.8a) and inverse inequalities for $a$ and $b$ to obtain

$$
\begin{aligned}
a & \lesssim \varepsilon_{1}\left(\frac{k_{1}^{2}}{h_{1}}\left\|\partial_{\theta} \partial_{\rho}^{2} \hat{u}\right\|_{L^{\infty}\left(\hat{\Omega}_{1}\right)}\|\hat{\varphi}\|_{L^{1}\left(\hat{\Omega}_{1}\right)}+\frac{k_{2}^{2}}{h_{2}}\left\|\partial_{\theta} \partial_{\rho}^{2} \hat{u}\right\|_{L^{\infty}\left(\hat{\Omega}_{2}\right)}\|\hat{\varphi}\|_{L^{1}\left(\hat{\Omega}_{2}\right)}\right) \\
& \lesssim \varepsilon_{1} N^{-1}(\ln N)^{2}\left(\varepsilon_{1}^{2}+\varepsilon_{2}^{2}\right)\left(1+\varepsilon_{1}^{-1}+\varepsilon_{2}^{-2}\right)\|\varphi\|_{0} \\
& \lesssim N^{-1}(\ln N)^{2}\left(\varepsilon_{1}+\varepsilon_{2}^{2}\right)\|\varphi\|_{0} \\
& \lesssim N^{-1}\|\varphi\|_{0}, \\
b & \lesssim \varepsilon_{1}\left(\frac{h_{1}^{2}}{k_{1}}\left\|\partial_{\theta}^{2} \partial_{\rho} \hat{u}\right\|_{L^{\infty}\left(\hat{\Omega}_{1}\right)}\|\hat{\varphi}\|_{L^{1}\left(\hat{\Omega}_{1}\right)}+\frac{h_{2}^{2}}{k_{2}}\left\|\partial_{\theta}^{2} \partial_{\rho} \hat{u}\right\|_{L^{\infty}\left(\hat{\Omega}_{2}\right)}\|\hat{\varphi}\|_{L^{1}\left(\hat{\Omega}_{2}\right)}\right) \\
& \lesssim \varepsilon_{1} \frac{N^{-1}}{\ln N}\left(\varepsilon_{1}^{-1}\left(\varepsilon_{1} \ln N\right)^{1 / 2}+\varepsilon_{2}^{-1}\left(\varepsilon_{2} \ln N\right)^{1 / 2}\right)\left(1+\varepsilon_{2}^{-1}\right)\|\varphi\|_{0} \\
& \lesssim \frac{N^{-1}}{(\ln N)^{1 / 2}}\left(\varepsilon_{2}+\varepsilon_{2}^{3 / 2}\right)\left(1+\varepsilon_{2}^{-1}\right)\|\varphi\|_{0} \\
& \lesssim N^{-1}\|\varphi\|_{0} .
\end{aligned}
$$

For $c$ and $d$ we use (3.8b). As we have periodicity in $\theta$-direction, we can ignore the second sum when bounding $d$. But then $d$ can be estimated by the same bound as $c$ due to exchanging 
$\rho$ and $\theta$ as well as $h$ and $k$. Therefore, it follows that

$$
\begin{aligned}
& c \lesssim \varepsilon_{1} \\
&\left(h_{1}\left\|\partial_{\theta}^{2} \partial_{\rho} \hat{u}\right\|_{L^{\infty}\left(\hat{\Omega}_{1}\right)}+k_{1}\left\|\partial_{\theta} \partial_{\rho}^{2} \hat{u}\right\|_{L^{\infty}\left(\hat{\Omega}_{1}\right)}\right)\left(\varepsilon_{1} \ln N\right)^{1 / 2}\|\varphi\|_{0} \\
&+\varepsilon_{1}\left(h_{2}\left\|\partial_{\theta}^{2} \partial_{\rho} \hat{u}\right\|_{L^{\infty}\left(\hat{\Omega}_{2}\right)}+k_{2}\left\|\partial_{\theta} \partial_{\rho}^{2} \hat{u}\right\|_{L^{\infty}\left(\hat{\Omega}_{2}\right)}\right)\left(\varepsilon_{2} \ln N\right)^{1 / 2}\|\varphi\|_{0} \\
&+\varepsilon_{1} h_{1}\left\|\partial_{\theta}^{2} \hat{u}\right\|_{L^{\infty}(\rho=0)}\|\varphi\|_{L^{1}(\rho=0)} \\
&+\varepsilon_{1} h_{2}\left\|\partial_{\theta}^{2} \hat{u}\right\|_{L^{\infty}\left(\rho=\lambda_{2}\right)}\|\varphi\|_{L^{1}\left(\rho=\lambda_{2}\right)} \\
& \lesssim \varepsilon_{1} N^{-1}\left(1+\varepsilon_{1}^{-1}+\varepsilon_{2}^{-2}+0+\left(1+N^{-\sigma_{1}}+N^{-\sigma_{2}}\right) N^{1 / 2}\right)\|\varphi\|_{0} \\
& \lesssim\left(N^{-1}+\varepsilon_{1} N^{-1 / 2}\right)\|\varphi\|_{0} \lesssim N^{-1}\|\varphi\|_{0}, \\
& d \lesssim
\end{aligned}
$$

Combining Lemmas 3.5 to 3.7 we obtain the main result of this section.

THEOREM 3.8. Let $(u, w)$ be the solution of (3.1) fulfilling Assumption 3.1 and $\left(u_{N}, w_{N}\right)$ be the solution of (3.2). Under the conditions $\sigma_{1} \geq 2, \sigma_{2} \geq 2+1 / 4$, and $\varepsilon_{1} \lesssim N^{-1}$, we have

$$
\left|\|\left(u-u_{N}, w-w_{N}\right)\right| \mid \lesssim N^{-1} \ln N .
$$

REMARK 3.9. The proof of Lemma 3.7 shows that Theorem 3.8 remains true under the conditions $\sigma_{1} \geq 2, \sigma_{2} \geq 2$, and $\varepsilon_{1} \lesssim N^{\frac{4}{3} \sigma_{2}-4}$.

4. Numerical experiments. In this section we present the results of numerical experiments. All computations were made using the finite element library $\mathbb{S O F} \mathbb{E}^{1}$ running in Matlab/Octave.

In our examples the exact solution is not known. Therefore, we use a numerically computed reference solution as substitute. This reference solution is computed on a Shishkin mesh twice as fine as the finest one used in our computations and with a polynomial degree of two.

We will present results in the energy norm

$$
\|(u, w)\|^{2}:=\|w\|_{0}^{2}+\varepsilon_{2}^{2}\|\nabla u\|_{0}^{2}+\left\|c_{0} u\right\|_{0}^{2} .
$$

This norm is not a balanced norm, i.e., for $\varepsilon_{1} \leq \varepsilon_{2} \rightarrow 0$ not all solution components contribute equally to it. With the decomposition $u=S+E_{1}+E_{2}$, where $S$ is the smooth part, $E_{1}$ are the faster decaying layers, and $E_{2}$ are the slower decaying layers, we have the estimates

$$
\begin{aligned}
\left\|\left(S, \varepsilon_{1} \Delta S\right)\right\|^{2} & \lesssim \varepsilon_{1}^{2}+\varepsilon_{2}^{2}+1 \sim 1, \\
\left\|\left(E_{1}, \varepsilon_{1} \Delta E_{1}\right)\right\|^{2} & \lesssim \frac{\varepsilon_{1}}{\varepsilon_{2}}+\frac{\varepsilon_{1}}{\varepsilon_{2}}+\frac{\varepsilon_{1}^{2}}{\varepsilon_{2}^{3}} \sim \frac{\varepsilon_{1}}{\varepsilon_{2}} \rightarrow 0, \\
\left\|\left(E_{2}, \varepsilon_{1} \Delta E_{2}\right)\right\|^{2} & \lesssim \frac{\varepsilon_{1}^{2}}{\varepsilon_{2}^{3}}+\varepsilon_{2}+\varepsilon_{2} \sim \varepsilon_{2} \rightarrow 0 .
\end{aligned}
$$

On the other hand, we obtain with

$$
\|(u, w)\|_{b}^{2}:=\frac{\varepsilon_{2}}{\varepsilon_{1}}\|w\|_{0}^{2}+\varepsilon_{2}\|\nabla u\|_{0}^{2}+\left\|c_{0} u\right\|_{0}^{2}
$$

${ }^{1}$ https://github.com/SOFE-Developers/SOFE 


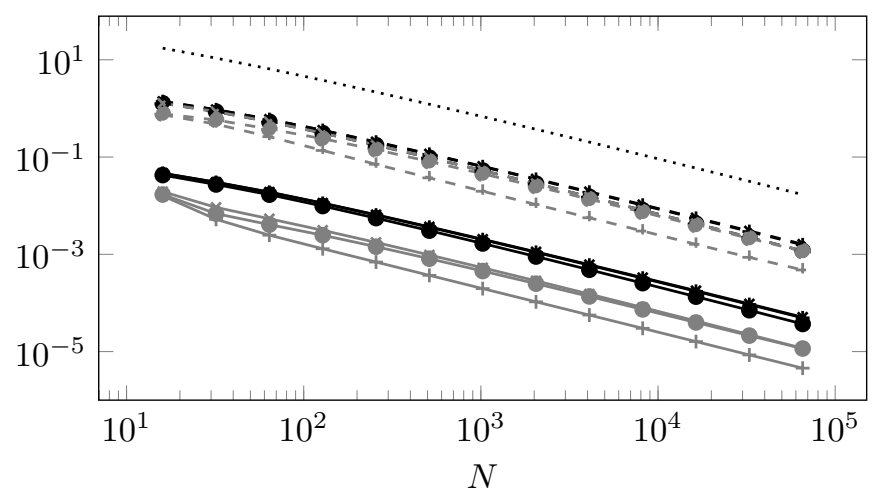

FIG. 4.1. Convergence behaviour in the energy norm (solid line) and the balanced norm (dashed line) for $\varepsilon_{1}=10^{-8}(\bullet), 10^{-9}(+)$, and $10^{-10}(\mathrm{x})$ and for $\varepsilon_{2}=10^{-3}$ (black) and $10^{-4}$ (gray).

a balanced norm with

$$
\begin{aligned}
\left\|\mid\left(S, \varepsilon_{1} \Delta S\right)\right\|_{b}^{2} & \lesssim \varepsilon_{1} \varepsilon_{2}+\varepsilon_{2}+1 \sim 1, \\
\left\|\mid\left(E_{1}, \varepsilon_{1} \Delta E_{1}\right)\right\|_{b}^{2} & \lesssim 1+\frac{\varepsilon_{1}}{\varepsilon_{2}^{2}}+\frac{\varepsilon_{1}^{2}}{\varepsilon_{2}^{3}} \sim 1, \\
\mid\left\|\left(E_{2}, \varepsilon_{1} \Delta E_{2}\right)\right\|_{b}^{2} & \lesssim \frac{\varepsilon_{1}}{\varepsilon_{2}^{2}}+1+\varepsilon_{2} \sim 1 .
\end{aligned}
$$

Unfortunately, the numerical method is not coercive with this norm and proving uniform convergence with respect to this stronger norm is an open problem. Nevertheless, we do present numerical results also with respect to this norm.

EXAMPLE 4.1. Let us start with a $1 \mathrm{D}$ problem given on $\Omega=(0,1)$. We simply set $c=1$ and $f(x)=1+\frac{\sin (8 x)}{2}$. The right-hand side is chosen such that it is not zero at the endpoints of $\Omega$ and not polynomial.

Figure 4.1 displays the convergence behaviour of our method for various values of $\varepsilon_{1}, \varepsilon_{2}$, and $N$. It can be seen that the errors in both the energy and the balanced norms behave like $N^{-1} \ln N$. This reference rate is depicted for comparison as a dotted line. Thus the theoretical result of Theorem 2.4 is confirmed numerically.

EXAMPLE 4.2. We consider a smooth domain $\Omega \subset \mathbb{R}^{2}$ with a boundary $\Gamma$ given by a curve $\gamma(\theta)$ using polar coordinates. We consider the so called Cranioid-curve with

$$
\gamma(\theta)=\left(\frac{1}{4} \sin (\theta)+\frac{1}{2} \sqrt{1-0.9 \cos (\theta)^{2}}+\frac{1}{2} \sqrt{1-0.7 \cos (\theta)^{2}}\right) \cdot\left[\begin{array}{l}
\cos (\theta) \\
\sin (\theta)
\end{array}\right]
$$

and $\theta \in[0,2 \pi)$. This domain together with a mesh for $N=16$ and rather large values of $\varepsilon_{1}$ and $\varepsilon_{2}$ is presented in Figure 3.1. On this domain $\Omega$ we choose $c=1$ and $f(x, y)=10 x$ as right-hand side.

The mesh is not a tensor-product mesh. Therefore, Table 4.1 presents the almost quadratic dependence of the number of degrees of freedom on $N$ for $\varepsilon_{1}=10^{-10}$ and $\varepsilon_{2}=10^{-4}$. For other values of $\varepsilon_{1}, \varepsilon_{2}$, the exact number of degrees of freedom changes only slightly.

Figure 4.2 displays the convergence behaviour of our method for various values of $\varepsilon_{1}, \varepsilon_{2}$, and $N$. It can be seen that also for the $2 \mathrm{D}$-problem the errors in both the energy and the balanced norm behave like $N^{-1} \ln N$, again depicted for comparison as a dotted line. Thus, the theoretical result of Theorem 3.8 is confirmed numerically. Furthermore, the balanced norm is larger than the energy norm (as expected), but for our example we still see a reduction in the errors for $\varepsilon_{2}$ becoming smaller. This is even more pronounced in the energy norm. 
TABLE 4.1

Quadratic dependence of the number of degrees of freedom (\#DoF) on $N$.

\begin{tabular}{r|r}
$N$ & \#DoF \\
\hline 8 & 102 \\
16 & 388 \\
32 & 1602 \\
64 & 6495 \\
128 & 25957 \\
256 & 105543 \\
512 & 426187
\end{tabular}

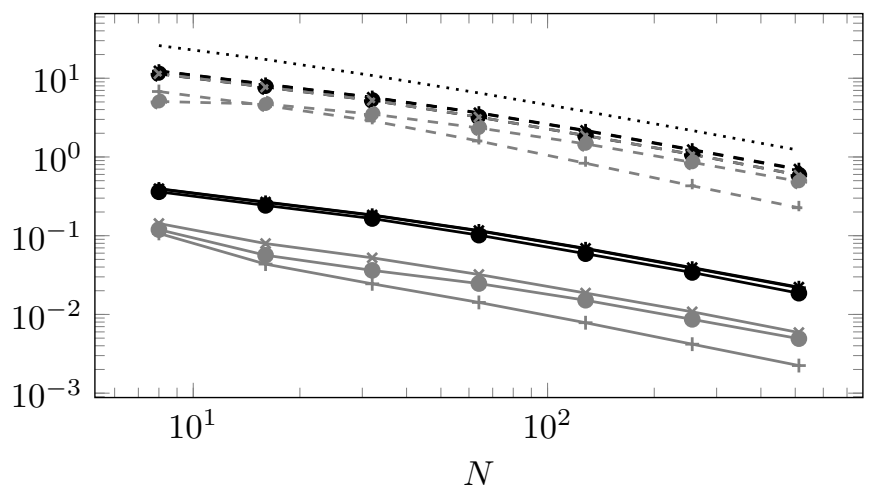

FIG. 4.2. Convergence behaviour in the energy norm (solid line) and the balanced norm (dashed line) for $\varepsilon_{1}=10^{-8}(\bullet), 10^{-9}(+)$, and $10^{-10}(\mathrm{x})$ and $\varepsilon_{2}=10^{-3}$ (black) and $10^{-4}$ (gray).

Acknowledgment. This paper was written during a visit by Mirjana Brdar to the Technical University of Dresden in December 2017-February 2018, supported by a DAAD grant, number 57314019, and partly supported by the Ministry of Science of the Republic of Serbia under grant no 44006.

\section{REFERENCES}

[1] H. BLUM AND R. RANNACHER, On the boundary value problem of the biharmonic operator on domains with angular corners, Math. Methods Appl. Sci., 2 (1980), pp. 556-581.

[2] P. Constantinou, S. Franz, L. Ludwig, And C. Xenophontos, A mixed hp fem for the approximation of $4^{\text {th }}$ order singularly perturbed problems on smooth domains, Numer. Methods Partial Differential Equations, 35 (2019), pp. 114-127.

[3] P. Constantinou, C. Varnava, And C. Xenophontos, An hp finite element method for $4^{\text {th }}$ order singularly perturbed problems, Numer. Algorithms, 73 (2016), pp. 567-590.

[4] S. FRANZ AND H.-G. RoOS, Robust error estimation in energy and balanced norms for singularly perturbed fourth order problems, Comput. Math. Appl., 72 (2016), pp. 233-247.

[5] S. FRANZ, H.-G. RoOS, AND A. WACHTEL, A C $\mathrm{C}^{0}$ interior penalty method for a singularly-perturbed fourth-order elliptic problem on a layer-adapted mesh, Numer. Methods Partial Differential Equations, 30 (2014), pp. 838-861.

[6] R. E. O’Malley, JR., Introduction to Singular Perturbations, Academic Press, New York, 1974.

[7] P. Panaseti, A. Zouvani, M. N., And C. Xenophontos, $A C^{1}$-conforming hp finite element method for fourth order singularly perturbed boundary value problems, Appl. Numer. Math., 104 (2016), pp. 81-97.

[8] H.-G. Roos, M. STynes, And L. ToBISKA, Robust Numerical Methods for Singularly Perturbed Differential Equations, 2nd ed., Springer, Berlin, 2008.

[9] B. SEMPER, Locking in finite-element approximations to long thin extensible beams, IMA J. Numer. Anal., 14 (1994), pp. 97-109. 


\section{ETNA}

Kent State University and

Johann Radon Institute (RICAM)

[10] R. SEvilla, S. FernándeZ-MÉndez, AND A. Huerta, NURBS-enhanced finite element method (NEFEM), Arch. Comput. Methods. Eng., 18 (2011), pp. 441-484.

[11] G. SHISHKIn, Grid approximation of singularly perturbed elliptic and parabolic equations, second doctorial thesis, Keldysh Institute, Moscow, 1990.

[12] _ Grid approximation of the solution and diffusion flux for singularly perturbed parabolic equations, in Proceedings of the 7th International Conference on Boundary and Interior Layers: Computational and Asymptotic Methods, Beijing, 1994, F. Zhuang, ed., Int. Academic Publishers, Beijing, 1995, pp. 12-19.

[13] M. STYNES AND L. TOBISKA, The SDFEM for a convection-diffusion problem with a boundary layer: optimal error analysis and enhancement of accuracy, SIAM J. Numer. Anal., 41 (2003), pp. 1620-1642.

[14] G. F. SUN AND M. STYNES, Finite-element methods for singularly perturbed high-order elliptic two-point boundary value problems. I. Reaction-diffusion-type problems, IMA J. Numer. Anal., 15 (1995), pp. 117139.

[15] N. YAn, Superconvergence Analysis and A Posteriori Error Estimation in Finite Element Methods, vol. 40 of Series in Information and Computational Science, Science Press, Beijing, 2008. 